\title{
EVN and MERLIN observations of microquasar candidates at low galactic latitudes
}

\author{
M. Ribó ${ }^{1}$, E. Ros ${ }^{2}$, J. M. Paredes ${ }^{1}$, M. Massi ${ }^{2}$, and J. Martî $\hat{i}^{3}$ \\ 1 Departament d'Astronomia i Meteorologia, Universitat de Barcelona, Av. Diagonal 647, 08028 Barcelona, Spain \\ 2 Max-Planck-Institut für Radioastronomie, Auf dem Hügel 69, 53121 Bonn, Germany \\ ${ }^{3}$ Departamento de Física, Escuela Politécnica Superior, Universidad de Jaén, Virgen de la Cabeza 2, 23071 Jaén, Spain
}

Received 2 August 2002 / Accepted 27 August 2002

\begin{abstract}
In an attempt to increase the number of known microquasars, Paredes et al. (2002) have presented a long-term project focused on the search for new objects of this type. They performed a cross-identification between X-ray and radio catalogs under very restrictive selection criteria for sources with $|b|<5^{\circ}$, and obtained a sample of 13 radio-emitting X-ray sources. Follow-up observations of 6 of these sources with the VLA provided accurate coordinates, which were used to discover optical counterparts for all of them. We have observed these six sources with the EVN and MERLIN at $5 \mathrm{GHz}$. Five of the six objects have been detected and imaged, presenting different morphologies: one source has a two-sided jet, three sources have one-sided jets, and one source is compact. With all the presently available information, we conclude that two of the sources are promising microquasar candidates in our Galaxy.
\end{abstract}

Key words. X-rays: binaries - radio continuum: stars

\section{Introduction}

Microquasars are stellar-mass black holes or neutron stars that mimic, on smaller scales, many of the phenomena seen in AGN and quasars. Microquasars have been found in X-ray binary systems, where a compact object accretes matter from a companion star. Radio Emitting X-ray Binaries (REXBs) with relativistic radio jets, like SS 433, GRS 1915+105, GRO J1655-40 or Cyg X-3, are good examples of microquasars (see Mirabel \& Rodríguez 1999 for a detailed review), while other well known sources like LS I +61303 could turn out to be new relativistic jet sources (Massi et al. 2002). With the recent addition of LS 5039 (Paredes et al. 2000), Cyg X-1 (Stirling et al. 2001) and XTE J1550-564 (Hannikainen et al. 2001) to the microquasar group, the current known number of this kind of sources is 14 , among $\sim 50$ REXBs within the $\sim 280$ known X-ray binaries (Liu et al. 2000; Liu et al. 2001). Recent studies of microquasars can be found in Castro-Tirado et al. (2001).

As pointed out in Sect. 1 of Paredes et al. (2002, hereafter Paper I), the number of known microquasars remains still small, especially when trying to study them from a statistical point of view. An interesting aspect of microquasars is their possibility of being related to unidentified high-energy $\gamma$ ray sources, as suggested by Paredes et al. (2000). Moreover, some parameters like the jet velocity, seem to be related to the mass of the compact object (i.e., with its potential well).

Send offprint requests to: M. Ribó,

e-mail: mribo@am.ub.es
Nevertheless, the lack of meaningful statistical studies because of the small population of microquasars with known jet velocities, prevents any definitive statements of this kind being made. Therefore, it is worth searching for new microquasars in order to increase the known population.

In this context, the sources to search for are REXBs, which are in fact microquasar candidates. To this end, a cross-identification between the X-ray ROSAT all sky Bright Source Catalog (RBSC) (Voges et al. 1999) and the NRAO VLA Sky Survey (NVSS) (Condon et al. 1998) was made for sources with $|b|<5^{\circ}$ under very restrictive selection criteria, and the obtained results have been presented in Paper I. A sample containing 13 sources was obtained, and 6 of them were observed at radio wavelengths in order to obtain radio spectra and their variability, as well as accurate radio positions, which allowed the authors to discover the corresponding optical counterparts for all of them. At the end of this study, two of the sources, namely 1RXS J001442.2+580201 and 1RXS J013106.4+612035 were classified as promising microquasar candidates. The remaining four sources were classified as weaker candidates for a number of reasons. 1RXS J042201.0+485610 shows a highly inverted spectrum at high radio frequencies and an optical counterpart slightly extended. 1RXS J062148.1+174736 is an object extended in the optical. 1RXS J072259.5-073131 presents a one-sided radio jet at arcsecond scales, supporting the possibility of being extragalactic. And finally, 1RXS J072418.3-071508 is a known quasar. 
Table 1. MERLIN positions for the five detected target sources, obtained via phase-referencing. The uncertainties quoted for the target sources were provided by the JMFIT task in AIPS (measuring in the phase-referenced image) and do not include systematic errors. The positions of all phase-reference calibrators except 1RXS J072418.3-071508 have an accuracy better than 1 mas.

\begin{tabular}{|c|c|c|c|c|c|}
\hline \multicolumn{3}{|c|}{ Target sources } & \multicolumn{3}{|c|}{ Phase-reference calibrators } \\
\hline 1RXS name & $\alpha(\mathrm{J} 2000.0)$ & $\delta(\mathrm{J} 2000.0)$ & Source name & $\alpha(\mathrm{J} 2000.0)$ & $\delta(\mathrm{J} 2000.0)$ \\
\hline J001442.2+580201 & $\begin{array}{r}00^{\mathrm{h}} 14^{\mathrm{m}} 42^{\mathrm{s}} .12822 \\
\pm 0.00013\end{array}$ & $\begin{array}{r}+58^{\circ} 02^{\prime} 01^{\prime \prime} 2460 \\
\pm 0 .{ }^{\prime \prime} 0022\end{array}$ & $\mathrm{~J} 0007+5706^{a}$ & $00^{\mathrm{h}} 07^{\mathrm{m}} 48^{\mathrm{s}} .47110$ & $+57^{\circ} 06^{\prime} 10^{\prime \prime} 4540$ \\
\hline J013106.4+612035 & $\begin{array}{r}01^{\mathrm{h}} 31^{\mathrm{m}} 07^{\mathrm{s}} .23210 \\
\pm 0.00014\end{array}$ & $\begin{array}{r}+61^{\circ} 20^{\prime} 33^{\prime \prime} 3752 \\
\pm 0^{\prime \prime} 0016\end{array}$ & $\mathrm{~J} 0147+5840^{a}$ & $01^{\mathrm{h}} 47^{\mathrm{m}} 46^{\mathrm{s}} .54380$ & $+58^{\circ} 40^{\prime} 44^{\prime \prime} 9750$ \\
\hline J062148.1+174736 & $\begin{array}{r}06^{\mathrm{h}} 21^{\mathrm{m}} 47^{\mathrm{s}} .75264 \\
\pm 0.00006\end{array}$ & $\begin{array}{r}+17^{\circ} 47^{\prime} 35^{\prime \prime} 0818 \\
\pm 0^{\prime \prime} 0017\end{array}$ & $\mathrm{~J} 0630+1738^{b}$ & $06^{\mathrm{h}} 30^{\mathrm{m}} 07^{\mathrm{s}} .25870$ & $+17^{\circ} 38^{\prime} 12^{\prime \prime} 9300$ \\
\hline J072259.5-073131 & $\begin{array}{r}07^{\mathrm{h}} 22^{\mathrm{m}} 59^{\mathrm{s}} .68188 \\
\pm 0.00011\end{array}$ & $\begin{array}{r}-07^{\circ} 31^{\prime} 34^{\prime \prime} 8009 \\
\pm 00^{\prime \prime} 0022\end{array}$ & 1RXS J072418.3-071508 ${ }^{c}$ & $07^{\mathrm{h}} 24^{\mathrm{m}} 17^{\mathrm{s}} .2912$ & $-07^{\circ} 15^{\prime} 20^{\prime \prime} 339$ \\
\hline $\mathrm{J} 072418.3-071508^{d}$ & $\begin{array}{r}07^{\mathrm{h}} 24^{\mathrm{m}} 17^{\mathrm{s}} .2912 \\
\pm 0.0007\end{array}$ & $\begin{array}{r}-07^{\circ} 15^{\prime} 20^{\prime \prime} 339 \\
\pm 00^{\prime \prime} 010\end{array}$ & J0730-116 & $07^{\mathrm{h}} 30^{\mathrm{m}} 19.1125$ & $-11^{\circ} 41^{\prime} 12^{\prime \prime} 601$ \\
\hline
\end{tabular}

a Position from Patnaik et al. (1992).

$b$ Position from Browne et al. (1998).

c This position was obtained from VLA observations (listed in the line below).

$d$ The entries in this line correspond to VLA observations (see Paper I).

In this paper we present Very Long Baseline Interferometry (VLBI) observations of these six sources, aimed at revealing possible jet-like features at milliarcsecond scales. We describe the observations and the data reduction in Sect. 2, present the results and a discussion in Sect. 3, and we summarize our findings in Sect. 4.

\section{Observations and data reduction}

We observed the six sources studied in Paper I simultaneously with the Multi-Element Radio-Linked Interferometer Network (MERLIN) and the European VLBI Network (EVN) on February 29th/March 1st 2000 (23:30-23:05 UT) at $5 \mathrm{GHz}$. Since some of the target radio sources were faint, we scheduled the observations introducing phase-reference calibrators with cycle times of around $7 \mathrm{~min}$ (compatible with the expected coherence times). Apart from the calibrators presented in Table 1, 4C 61.02 was used as calibrator for 1RXS J013106.4+612035, and TXS 0422+496 and NRAO 150 for 1RXS J042201.0+485610. We also observed the fringefinder DA 193 and the MERLIN flux density calibrator 3C 286. Single dish flux density measurements were carried out with the MPIfR $100 \mathrm{~m}$ antenna in Effelsberg, Germany.

\subsection{MERLIN}

MERLIN is a connected radio interferometer across England, with baselines reaching up to $217 \mathrm{~km}$ length. This array observed with 2-bit sampling at dual polarisation with two blocks of 16 channels, each channel of $1 \mathrm{MHz}$ bandwidth. We analysed the left hand circular polarisation data excluding one channel at both edges of the band, yielding a final bandwidth of $14 \mathrm{MHz}$. The correlator integration time was of $4 \mathrm{~s}$.
The MERLIN data reduction was carried out at Jodrell Bank Observatory, using standard procedures within the Astronomical Image Processing System (AIPS, developed and maintained by the US National Radio Astronomy Observatory). We did not detect 1RXS J042201.0+485610. All other sources were detected, and accurate positions for the target radio sources were obtained via phase-referencing. These positions, presented in Table 1, were used later as a priori information for the VLBI correlation. The position given in Table 1 for the quasar 1RXS J072418.3-071508 was obtained from the VLA observations presented in Paper I. The position of 1RXS J072259.5-073131 is deduced from the phasereference offset relative to 1RXS J072418.3-071508 provided by MERLIN.

To image the sources, we averaged the data in frequency and exported them to be processed into the difference mapping software DIFMAP (Shepherd et al. 1994), where we timeaveraged the data in $32 \mathrm{~s}$ bins after careful editing.

\section{2. $E V N$}

The EVN observations were performed with the following array (name, code, location, diameter): Effelsberg, EB, Germany, 100 m; Jodrell Bank, JB, UK, 25 m; Cambridge, CM, UK, $32 \mathrm{~m}$; Westerbork, WB, The Netherlands, $14 \times 25 \mathrm{~m}$; Medicina, MC, Italy, 32 m; Noto, NT, Italy, 32 m; Shanghai, SH, China, $25 \mathrm{~m}$; Toruń, TR, Poland, $32 \mathrm{~m}$; and Onsala85, ON, Sweden, $25 \mathrm{~m}$. Data were recorded in MkIV mode with 2-bit sampling at $256 \mathrm{Mbps}$ with left hand circular polarization. A bandwidth of $64 \mathrm{MHz}$ was used, divided into 8 intermediate frequency (IF) bands.

The data were processed at the EVN MkIV correlator at the Joint Institute for VLBI in Europe (JIVE), in Dwingeloo, The Netherlands. The correlator integration time was of $4 \mathrm{~s}$. 
A first post-processing analysis was also carried out at JIVE. The data were processed using AIPS. A first a priori visibility amplitude calibration was performed using antenna gains and system temperatures measured at each antenna. The fringe fitting (FRING) of the residual delays and fringe rates was performed for all the radio sources. No fringes were found for 1RXS J042201.0+485610 and 4C 61.02. Fringes for many baselines were missing for 1RXS J001442.2+580201, 1RXS J062148.1+174736 and TXS 0422+496.

To improve the fringe detection on all baselines for 1RXS J001442.2+580201 and 1RXS J062148.1+174736 we used the delay, rate, and phase solutions from their corresponding phase-reference calibrators $\left(\mathrm{J} 0007+5706,1^{\circ} 18^{\prime}\right.$ separation, and $\mathrm{J} 0630+1738,1^{\circ} 59^{\prime}$ separation) and interpolated them to the target sources using the AIPs task CLCAL. We fringefitted the target sources again using narrower search windows and obtained solutions for all baselines. A similar attempt on 1RXS J042201.0+485610 (with respect to TXS 0422+486 and NRAO 150) was unfruitful. Effelsberg was used as reference antenna throughout the AIPS data reduction process.

We then averaged the data in frequency and exported them to be imaged and self-calibrated in DIFMAP. The a priori visibility amplitude calibration was not sufficient to reliably image the weakest radio sources. We improved that by first imaging in DIFMAP the calibrator sources J0007+5706, J0147+5840, and $\mathrm{J} 0630+1738$, with appropriate amplitude self-calibration. We deduced correction factors for each antenna, these being consistent for the three radio sources within $2 \%$. We corrected the amplitude calibration back in AIPs and exported the data again into DIFMAP, where the final imaging was performed after editing and averaging of the visibilities in $32 \mathrm{~s}$ blocks.

\subsection{Combining EVN and MERLIN}

The EVN and MERLIN arrays have one common baseline, between JB and CM, which allows to combine both data sets and map them together. We processed the data within AIPS in order to combine both arrays. The B1950.0 $(u, v)$ coordinates of the MERLIN data had to be corrected to the ones of the EVN for the same reference system (J2000.0) with UVFIX. Then, the MERLIN data were self-calibrated with the EVN images (see below), and the phase solutions were limited to the longest MERLIN baselines. The MERLIN data were imaged and the peak-of-brightness of both data sets were checked to be similar. As a next step, the EVN data were averaged in frequency to correspond to the MERLIN data. The AIPs headers of both data sets were modified conveniently to match together, and the weighting of both data sets was also modified to be equal with WTMOD. Finally, both data sets were concatenated (using DBCON) and exported to DIFMAP to be imaged with different data weighting in $(u, v)$ distance (tapering) after time averaging in 32 s bins.

\subsection{Flux density measurements at the $100 \mathrm{~m}$ antenna in Effelsberg}

We interleaved cross-scans (in azimuth and elevation) with the $100 \mathrm{~m}$ Effelsberg antenna to measure the radio source flux densities (A. Kraus, private communication). We fitted a Gaussian function to the flux-density response for every crossscan, and we averaged the different Gaussians. We linked the flux density scale by observing primary calibrators such as 3C 286, 3C 48, or NGC 7027 (see e.g. Kraus 1997; Peng et al. 2000). We list the single dish flux density measurements in the second column of Table 2. The flux density values for the main VLBI calibrators were of 7.5 $\pm 0.1 \mathrm{Jy}$ for $3 \mathrm{C} 286$, and $5.9 \pm 0.2 \mathrm{Jy}$ for DA 193.

\section{Results and discussion}

We present all the imaging results in Figs. 1-5, and the image parameters in Table 2 . The total flux density values for the different images diverge from each other and from the single dish measurements, due to the amplitude self-calibration process in all cases. Therefore, those values should be considered with care. The minimum contours in the images are those listed as $S_{\min }$ in Table 2, while consecutive higher contours scale with $3^{1 / 2}$. Here follows a detailed discussion on each source.

\subsection{RXS J001442.2+580201 and its two-sided jet}

As can be seen in our images, shown in Fig. 1, this source appears point-like at MERLIN resolution, partially resolved in the tapered EVN+MERLIN image and clearly resolved at EVN scales. In this last case, it shows a two-sided jet-like structure roughly in the north-south direction, with brighter components towards the south. The trends are visible in the closure phases, giving us confidence that the structure observed is not a consequence of sidelobes or imaging artifacts. In the tapered EVN+MERLIN images, the structure extends up to 20-30 mas outside of the core, and more clearly towards the north. This discrepancy could be due to calibration problems.

Model fitting of the EVN visibilities with circular Gaussians provides a parametrization of the inner structure. Five components reproduce the visibilities. The central one has $7.8 \mathrm{mJy}$, with a FWHM of 0.4 mas. Towards the north, one component (N2) of $0.6 \mathrm{mJy}$ at $3.6 \mathrm{mas}\left(\mathrm{PA}-10^{\circ}, F W H M 0.7 \mathrm{mas}\right)$ and another one $(\mathrm{N} 1)$ of $0.5 \mathrm{mJy}$ at $8.6 \mathrm{mas}$ (PA $2^{\circ}$, extended over 3 mas) are needed. Brighter components are present southwards, one (S2) of $1.1 \mathrm{mJy}$ at 5.0 mas (PA $177^{\circ}, F W H M$ of $0.8 \mathrm{mas})$ and the other one (S1) of $0.4 \mathrm{mJy}$ at $13.7 \mathrm{mas}$ (PA $177^{\circ}, F W H M$ below 0.3 mas).

If we assume that components $\mathrm{S} 1$ and $\mathrm{N} 1$ correspond to a pair of plasma clouds ejected at the same epoch near the compact object and perpendicularly to the accretion disk, we can estimate some parameters of the jets by using the following equation:

$\beta \cos \theta=\frac{\mu_{\mathrm{a}}-\mu_{\mathrm{r}}}{\mu_{\mathrm{a}}+\mu_{\mathrm{r}}}=\frac{d_{\mathrm{a}}-d_{\mathrm{r}}}{d_{\mathrm{a}}+d_{\mathrm{r}}}$,

$\beta$ being the velocity of the clouds in units of the speed of light, $\theta$ the angle between the direction of motion of the ejecta and the line of sight and $\mu_{\mathrm{a}}$ and $\mu_{\mathrm{r}}$ the proper motions of the approaching and receding components, respectively (Mirabel \& Rodríguez 1999). Although we do not know the epoch of ejection of the clouds, we can cancel the time variable by using the 
Table 2. Flux densities and parameters used to produce the images in Figs. 1-5.

\begin{tabular}{|c|c|c|c|c|c|c|c|}
\hline 1RXS name & $\begin{array}{c}\text { Single dish } \\
S_{\mathrm{EB}} \\
{[\mathrm{mJy}]}\end{array}$ & $\begin{array}{c}\text { Array }^{a} \\
\text { (taper } F W H M) \\
{[\mathrm{M} \lambda]}\end{array}$ & $\begin{array}{c}\text { beam size } \\
{[\mathrm{mas}] \times[\mathrm{mas}]}\end{array}$ & $\begin{array}{l}\mathrm{PA} \\
{\left[{ }^{\circ}\right]}\end{array}$ & $\begin{array}{c}S_{\text {tot }} \\
{[\mathrm{mJy}]}\end{array}$ & $\begin{array}{c}S_{\text {peak }} \\
{\left[\mathrm{mJy} \mathrm{beam}^{-1}\right]}\end{array}$ & $\begin{array}{c}S_{\min } \\
{\left[\mathrm{mJy} \mathrm{beam}^{-1}\right]}\end{array}$ \\
\hline \multirow[t]{3}{*}{$\mathrm{J} 001442.2+580201$} & $6.5 \pm 0.5^{b}$ & $\mathrm{M}$ & $57 \times 51$ & 37 & 6.2 & 5.8 & 0.1 \\
\hline & & $\mathrm{E}+\mathrm{M}(10)$ & $7.7 \times 7.2$ & -4 & 10.1 & 9.9 & 0.18 \\
\hline & & E & $1.77 \times 0.86$ & -20 & 11.5 & 7.0 & 0.15 \\
\hline \multirow[t]{3}{*}{ J013106.4+612035 } & $20.1 \pm 0.6$ & M & $71 \times 39$ & -63 & 17.5 & 17.9 & 0.5 \\
\hline & & $E+M(15)$ & $7.8 \times 6.2$ & -75 & 19.2 & 18.7 & 0.8 \\
\hline & & E & $1.04 \times 0.99$ & -1 & 17.6 & 11.6 & 0.4 \\
\hline $\mathrm{J} 042201.0+485610$ & $<5$ & - & - & - & - & - & - \\
\hline \multirow[t]{3}{*}{ J062148.1+174736 } & $7.1 \pm 0.7^{b}$ & M & $88 \times 39$ & 24 & 5.8 & 5.6 & 0.2 \\
\hline & & $\mathrm{E}+\mathrm{M}(8)$ & $13.1 \times 11.1$ & -24 & 6.0 & 6.8 & 0.3 \\
\hline & & E & $8.8 \times 4.1$ & 47 & 7.0 & 6.4 & 0.3 \\
\hline \multirow[t]{3}{*}{ J072259.5-073131 } & $67.9 \pm 1.1$ & $\mathrm{M}$ & $115 \times 66$ & 4 & 66.0 & 62.9 & 0.7 \\
\hline & & $\mathrm{E}+\mathrm{M}(15)$ & $9.5 \times 7.3$ & -61 & 52.1 & 41.9 & 0.9 \\
\hline & & E & $5.74 \times 1.12$ & 11 & 46.9 & 36.2 & 0.9 \\
\hline \multirow[t]{3}{*}{ J072418.3-071508 } & $282.2 \pm 4.1$ & M & $120 \times 64$ & 8 & 301.0 & 285.7 & 0.9 \\
\hline & & $\mathrm{E}+\mathrm{M}(10)$ & $11.7 \times 9.8$ & -61 & 287.0 & 263.4 & 2.0 \\
\hline & & E & $5.73 \times 1.02$ & 11 & 248.0 & 184.8 & 0.7 \\
\hline
\end{tabular}

${ }^{a}$ M: MERLIN. E+M: EVN+MERLIN, FWHM of the tapering function (weighting of visibilities) in parenthesis. E: EVN.

$b$ Values with low SNR in the Gaussian fits.
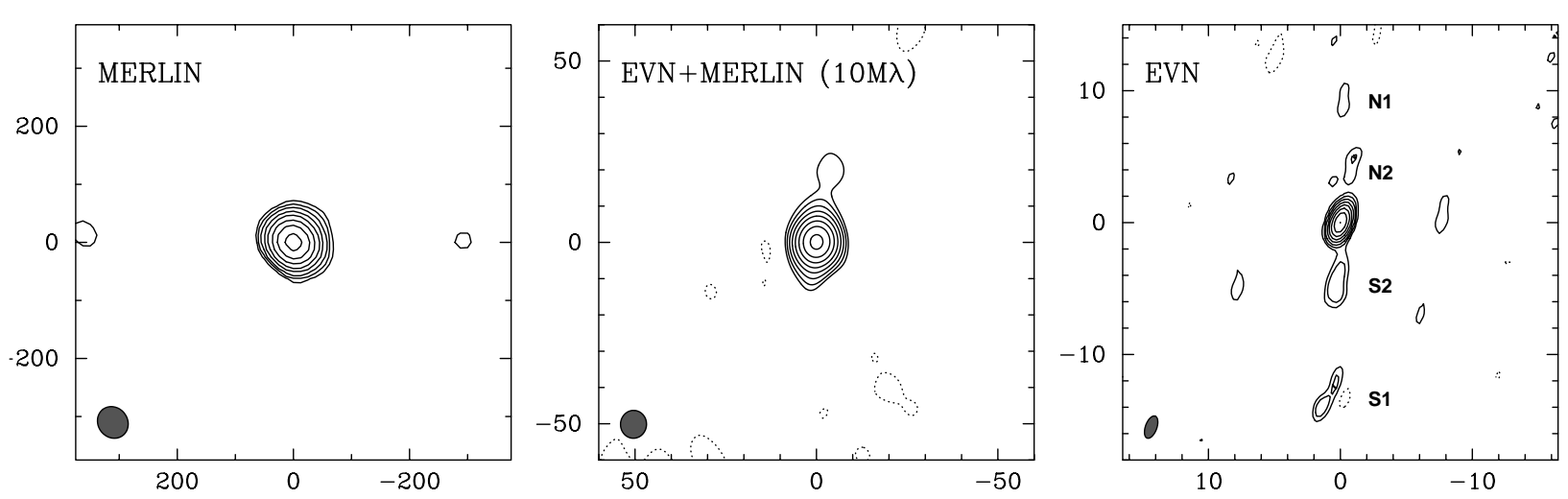

Fig. 1. Images of 1RXS J001442.2+580201 using the different arrays and with the parameters given in Table 2. The axes units are in mas. A $(u, v)$-tapering with a $F W H M$ at $10 \mathrm{M} \lambda$ has been used to perform the combined EVN+MERLIN image. In the EVN image, N1, N2, S2 and $\mathrm{S} 1$ indicate the components discussed in the text.

relative distances to the core $d_{\mathrm{a}}$ and $d_{\mathrm{r}}$, as expressed in Eq. (1). Since both variables, $\beta$ and $\cos \theta$, take values between 0 and 1 , it is clear that knowing $\beta \cos \theta$ allows us to compute a lower limit for the velocity $\left(\beta_{\min }\right)$ and an upper limit for the angle $\left(\theta_{\max }\right)$. The same applies for the S2 and N2 components. In Table 3 we list the positions of the components obtained from model fitting, together with the derived values from $\beta_{\min }$ and $\theta_{\max }$ for each one of the pairs. The slightly different results obtained using pair 1 or 2 , could be due to the fact that the position for the $\mathrm{S} 1$ component obtained with model fitting happens to be at the lower part of this elongated component, hence increasing $\beta \cos \theta$, or to intrinsic different velocities for each one of the pairs. Hereafter we will use $\beta>0.20 \pm 0.02$ and $\theta<78 \pm 1^{\circ}$.
Table 3. Model fitted positions of the components in the 1RXS J001442.2+580201 radio jets and obtained jet parameters.

\begin{tabular}{ccccc}
\hline \hline Comp. & $\begin{array}{c}\text { Distance } \\
{[\mathrm{mas}]}\end{array}$ & $\begin{array}{c}\text { PA } \\
{\left[{ }^{\circ}\right]}\end{array}$ & $\beta_{\min }$ & $\begin{array}{c}\theta_{\max } \\
{\left[{ }^{\circ}\right]}\end{array}$ \\
\hline $\mathrm{N} 1$ & $8.6 \pm 0.3$ & 2 & $0.23 \pm 0.02$ & $77 \pm 1$ \\
$\mathrm{~N} 2$ & $3.6 \pm 0.2$ & -10 & $0.16 \pm 0.02$ & $81 \pm 1$ \\
$\mathrm{~S} 2$ & $5.0 \pm 0.1$ & 177 & $0.16 \pm 0.02$ & $81 \pm 1$ \\
$\mathrm{~S} 1$ & $13.7 \pm 0.3$ & 177 & $0.23 \pm 0.02$ & $77 \pm 1$ \\
\hline
\end{tabular}



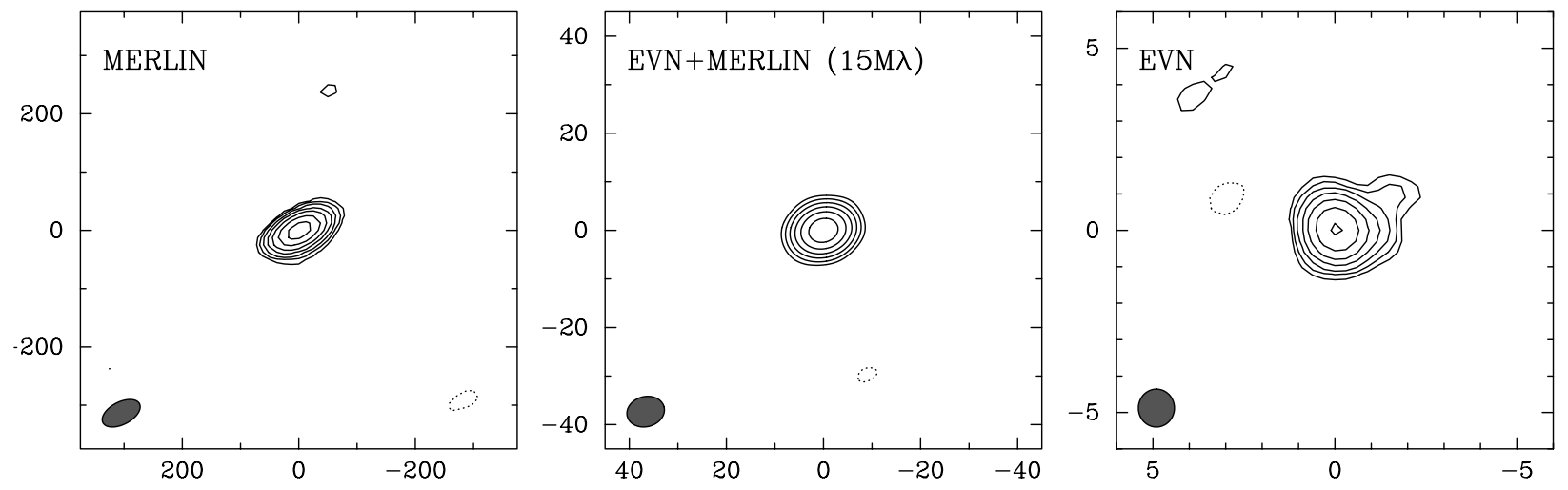

Fig. 2. Images of 1RXS J013106.4+612035 using the different arrays and with the parameters given in Table 2. The axes units are in mas. A $(u, v)$-tapering with a $F W H M$ at $15 \mathrm{M} \lambda$ has been used to perform the combined EVN+MERLIN image.

A similar approach to obtain the jet parameters of the source can be performed thanks to the brightness asymmetry of the components using the following equation (Mirabel \& Rodríguez 1999):

$\beta \cos \theta=\frac{\left(S_{\mathrm{a}} / S_{\mathrm{r}}\right)^{1 /(k-\alpha)}-1}{\left(S_{\mathrm{a}} / S_{\mathrm{r}}\right)^{1 /(k-\alpha)}+1}$,

where $S_{\mathrm{a}}$ and $S_{\mathrm{r}}$ are the flux densities of the approaching and receding components, respectively, $k$ equals 2 for a continuous jet and 3 for discrete condensations, and $\alpha$ is the spectral index of the emission $\left(S_{v} \propto v^{+\alpha}\right)$. However, the equation above is only valid when the components are at the same distance from the core. If this is not the case (i.e., $d_{\mathrm{r}}<d_{\mathrm{a}}$ ), the ratio $S_{\mathrm{a}} / S_{\mathrm{r}}$ will be lower than the one that should be used in Eq. (2) (because the flux density decreases with increasing distance from the core). In consequence, Eq. (2) only allows us to obtain a lower limit for $\beta \cos \theta$.

In order to use this approach we will consider $k=3$, because the components seem to be discrete condensations, and $\alpha=-0.20 \pm 0.05$, according to the overall spectral index reported in Paper I, since we do not have spectral index information of the components. The use of the flux densities obtained after model fitting gives $\beta \cos \theta>0.09 \pm 0.04$ for the $\mathrm{S} 2-\mathrm{N} 2$ pair and $\beta \cos \theta>-0.07 \pm 0.08$ for the $\mathrm{S} 1-\mathrm{N} 1$ pair. This last value is certainly surprising, although it can be explained by the fact that the S1 flux density obtained after model fitting does not account for the total flux of this plasma cloud. In fact, better estimates of the flux densities can be obtained summing together the flux densities of the CLEAN components obtained within each one of the four plasma clouds. This yields to $\beta \cos \theta>0.13 \pm 0.05$ for the $\mathrm{S} 2-\mathrm{N} 2$ pair and $\beta \cos \theta>0.17 \pm 0.02$ for the $\mathrm{S} 1-\mathrm{N} 1$ pair, in good agreement with the values computed using the distances from the components to the core, shown in Table 3.

If we compare the VLA position reported in Paper I with the MERLIN position in Table 1 we can see that they are different. In fact, taking into account the errors, the MERLIN-VLA position offsets can be expressed as: $\Delta \alpha \cos \delta=16 \pm 10$ mas and $\Delta \delta=27 \pm 10$ mas. Assuming that the difference in position is due to intrinsic proper motions and considering the time span between both observations, 224 days, we obtain: $\mu_{\alpha \cos \delta}=26 \pm 16 \mathrm{mas} \mathrm{yr}^{-1}$ and $\mu_{\delta}=44 \pm 16 \mathrm{mas} \mathrm{yr}^{-1}$. Although a proper motion of the source would clearly indicate its microquasar nature, because no proper motion would be detected in an extragalactic source, we must be cautious with this result. First of all, the phase-reference sources where different in the VLA and MERLIN observations, as well as the observing frequencies from which positions were estimated. On the other hand, none of these results exceeds the $3 \sigma$ value, preventing to state that a proper motion has been detected.

Summarizing, our results indicate that this source exhibits relativistic radio jets with $\beta>0.20$ and, therefore, together with the results reported in Paper I, we consider 1 RXS J001442.2+580201 as a very promising microquasar candidate.

\subsection{RXS J013106.4+612035 and its one-sided jet}

We show in Fig. 2 the images obtained after our observations. Although the source appears compact at MERLIN and EVN+MERLIN scales, there is a weak one-sided radio jet towards the northwest in the EVN image. Model fitting with circular Gaussian components can reproduce the observed visibilities as follows: a central $15.4 \mathrm{mJy}$ component with 0.64 mas $F W H M$, and a northwest $2.1 \mathrm{mJy}$ component with a $F W H M$ of 0.74 mas, located at 1.8 mas in $\mathrm{PA}-73^{\circ}$. As can be seen, this last component is located at a distance of $\sim 2$ times the beam size from the core.

Using the fact that we do not detect a counter-jet, we can use Eq. (2) replacing $S_{\mathrm{r}}$ with the $3 \sigma$ level value. This, of course, will only provide a lower limit to $\beta \cos \theta$, expressed as follows:

$\beta \cos \theta>\frac{\left(S_{\mathrm{a}} / 3 \sigma\right)^{1 /(k-\alpha)}-1}{\left(S_{\mathrm{a}} / 3 \sigma\right)^{1 /(k-\alpha)}+1}$

Using $S_{\mathrm{a}}=2.1 \mathrm{mJy}, 3 \sigma=0.30 \mathrm{mJy}$ (the $1 \sigma$ value has been taken as the root mean square noise in the image), $\alpha=$ $-0.05 \pm 0.05$ (see Paper I), and $k=3$ to be consistent with the lowest limit, we obtain $\beta \cos \theta>0.31 \pm 0.05(\beta>0.31 \pm 0.05$ and $\theta<72 \pm 3$ ). Hence, a lower limit of $\beta \geq 0.3$ is obtained, pointing towards relativistic radio jets as the origin of the 

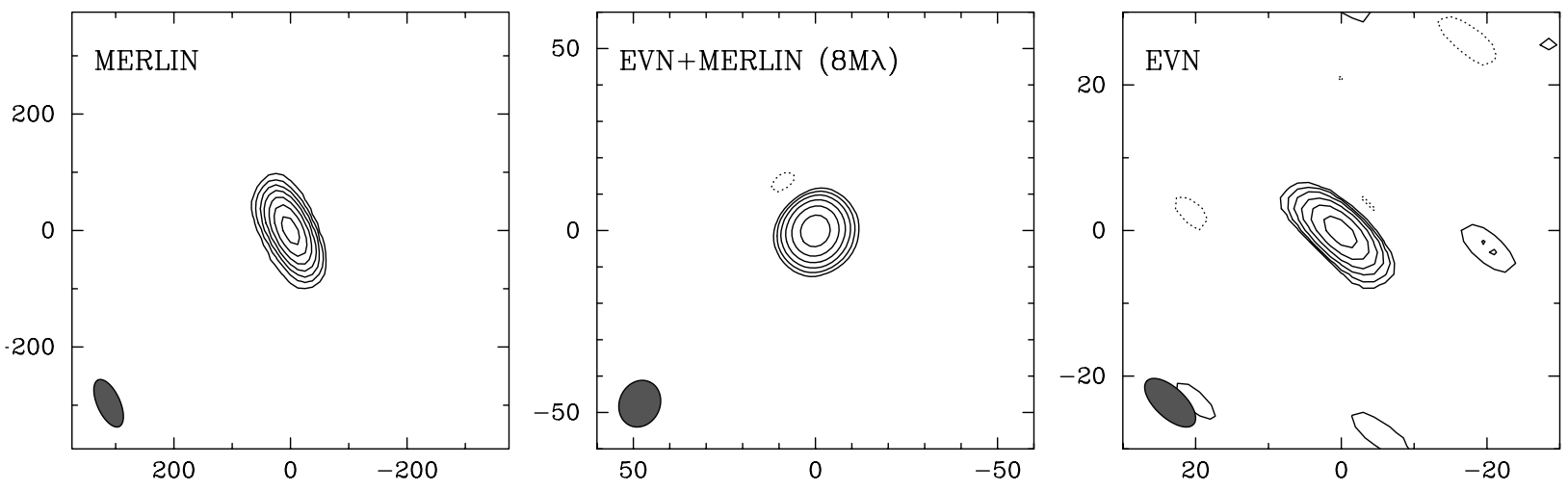

Fig. 3. Images of 1RXS J062148.1+174736 using the different arrays and with the parameters given in Table 2 . The axes units are in mas. A $(u, v)$-tapering with a $F W H M$ at $8 \mathrm{M} \lambda$ has been used to perform the combined EVN+MERLIN image.

elongated radio emission present in the EVN image. Although the one-sided jet morphology at mas scales is found mostly in extragalactic sources, it is also present in some galactic REXBs, like Cyg X-3 (Mioduszewski et al. 2001) or LS I +61 303 (Massi et al. 2001). Hence, we cannot rule out a possible galactic nature on the basis of the detected morphology.

As done for the previous source, we can compare the VLA position with the MERLIN one, and find that they differ in $\Delta \alpha \cos \delta=39 \pm 10$ mas and $\Delta \delta=-0.8 \pm 10$ mas. If the offsets are real, this would imply $\mu_{\alpha \cos \delta}=64 \pm 16{\text { mas } \mathrm{yr}^{-1}}^{-1}$ and $\mu_{\delta}=-1 \pm 16$ mas $\mathrm{yr}^{-1}$. Hence, it seems possible that we have detected a proper motion in right ascension at a $4 \sigma$ level. However, we must be cautious since, as in the previous case, the phase-reference sources and observing frequencies were different in each observation.

Overall, these results are indicative of relativistic radio jets, and hence, together with the results reported in Paper I, allow us to classify this source as a promising microquasar candidate.

\subsection{RXS J042201.0+485610, a non-detected source}

This radio source was marginally seen in the cross-scans carried out in Effelsberg. In fact, this is compatible with the low flux density of $2.3 \pm 0.4 \mathrm{mJy}$ at $1.4 \mathrm{GHz}$ listed in the NVSS. The source was not detected with MERLIN or with the EVN, mainly due to problems with the phase-reference sources, as pointed out in Sect. 2.2. In fact, as discussed in Paper I, VLA A configuration observations showed a flux density of $0.4 \mathrm{mJy}$ at $5 \mathrm{GHz}$ and an inverted spectrum with a spectral index up to $\alpha=+1.6$, suggesting thermal radio-emission resolved at higher angular resolutions. However, we cannot exclude the possibility of having a highly variable source.

\subsection{RXS J062148.1+174736, a compact source}

As can be seen in our images, shown in Fig. 3, the radio source is compact on all scales. In fact, model fitting of the EVN visibilities converges to a point-like radio source (the FWHM of a circular Gaussian tends to zero). We must note that when this source was observed it was below the horizon in $\mathrm{SH}$. Therefore, the obtained beam size for the EVN image is larger than the ones obtained for the other sources, as can be seen in Table 2, hence providing lower angular resolution than in the other cases. A comparison between the VLA and MERLIN positions reveals that they are perfectly compatible within the errors, suggesting an extragalactic nature or a small proper motion if it turns out to be a galactic microquasar. Although the compactness of the source is not indicative of a galactic or an extragalactic origin, the extended optical counterpart, reported in Paper I, suggests an extragalactic origin for this source. In fact, the detected radio variability from 8 to $11 \mathrm{mJy}$ within a week reported in Paper I, is compatible with the compactness in an extragalactic object (see IDV phenomenon, Wagner \& Witzel 1995).

\subsection{RXS J072259.5-073131 and its bent one-sided jet}

The images obtained at different resolutions are plotted in Fig. 4. The MERLIN image presents a compact structure with some elongation eastwards, while the EVN and combined EVN+MERLIN images show a clear one-sided jet towards the east, with a slight bent towards the south at larger core separations. The closure phases clearly show that the source departs from symmetry, with preferred emission to the east, both in the MERLIN and the EVN data sets. Two distinct components are present in the EVN image, at 9 and 17 mas from the compact core (PA of 89 and $113^{\circ}$, respectively). Those components can be model fitted with elliptical Gaussians, yielding flux densities of 3.7 and $2.8 \mathrm{mJy}$, respectively, for a core of $40.3 \mathrm{mJy}$.

Using again the fact that we do not detect a counter-jet, we can use Eq. (3) with $S_{\mathrm{a}}=3.7 \mathrm{mJy}$ (the closest component to the core in the EVN image), $3 \sigma=0.54 \mathrm{mJy}$ (as previously done, the $1 \sigma$ value has been taken as the root mean square noise in the image), $\alpha=-0.25 \pm 0.2$ (see Paper I), and $k=3$ to be consistent with the discrete nature of the components, to obtain $\beta \cos \theta>0.29 \pm 0.05$, and hence $\beta>0.29 \pm 0.05$ and $\theta<73 \pm 3^{\circ}$. 

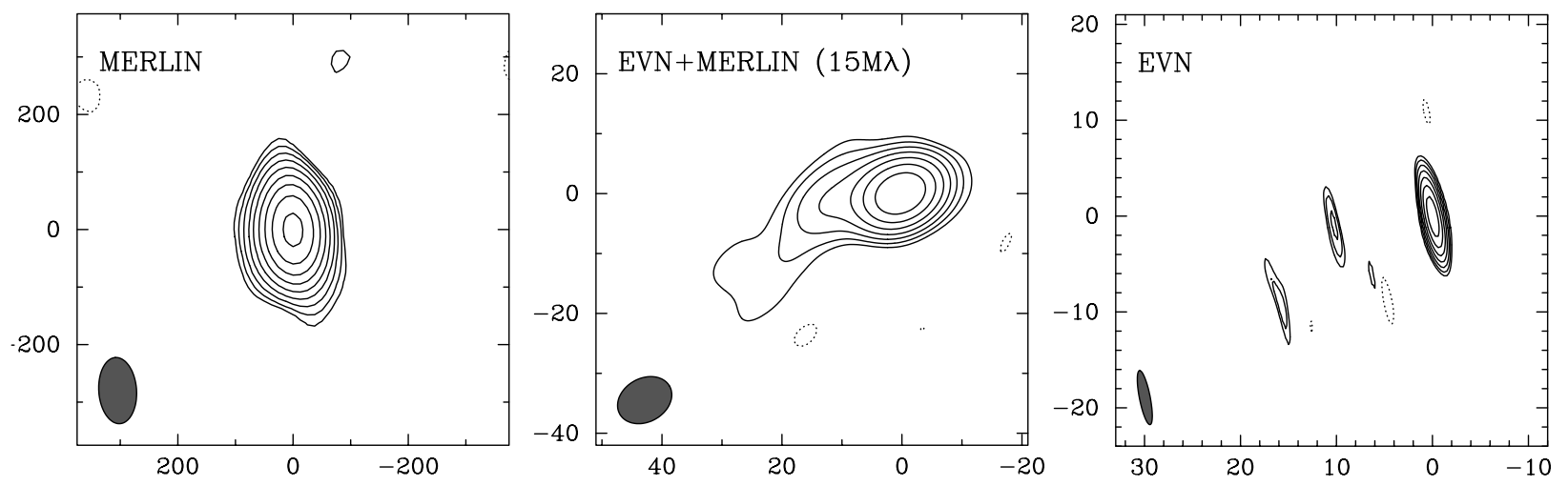

Fig. 4. Images of 1RXS J072259.5-073131 using the different arrays and with the parameters given in Table 2. The axes units are in mas. A $(u, v)$-tapering with a $F W H M$ at $15 \mathrm{M} \lambda$ has been used to perform the combined EVN+MERLIN image.
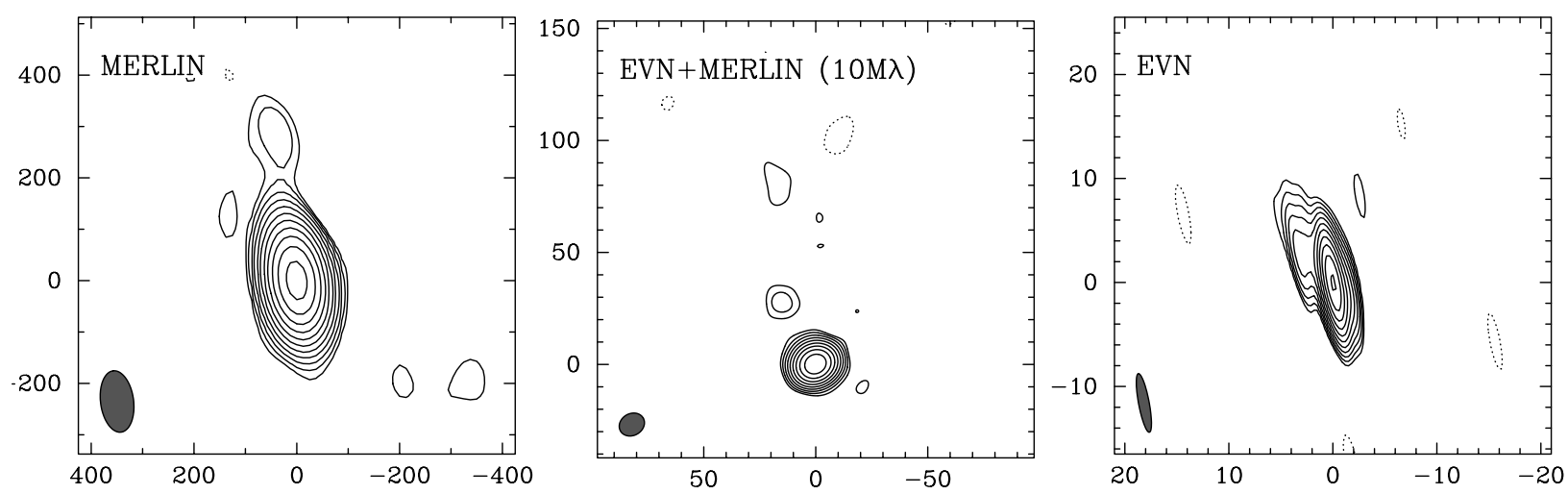

Fig. 5. Images of the already identified quasar 1RXS J072418.3-071508 using the different arrays and with the parameters given in Table 2 . The axes units are in mas. A $(u, v)$-tapering with a $F W H M$ at $10 \mathrm{M} \lambda$ has been used to perform the combined EVN+MERLIN image.

Therefore, these results point towards relativistic radio jets as the origin of the elongated radio emission present in the images.

As pointed out for 1RXS J013106.4+612035, the one-sided jet morphology does not rule out a microquasar nature for this object. However, the bending of the jet at such small angular scales resembles the ones seen in blazars. In fact, as reported in Paper I, a one-sided arcsecond scale jet is also present in VLA A configuration observations at $1.4 \mathrm{GHz}$, an unusual feature in the already known microquasars.

A comparison between the VLA and MERLIN positions reveals that they agree within the errors, suggesting an extragalactic nature or a small proper motion if it turns out to be a galactic microquasar.

Overall, these results are indicative of relativistic radio jets, although this source shows characteristics more similar to blazars than to microquasars.

\subsection{RXS J072418.3-071508, a quasar with a bent one-sided jet}

This radio source has recently been (March 2002) classified as a quasar in the SIMBAD database, and it is not any more a microquasar candidate. It is catalogued in the Parkes-MIT-NRAO
(Griffith et al. 1994) and the Texas Survey (Douglas et al. 1996). It is listed as PMN J0724-0715 in the NED database, and is the source WGA J0724.3-0715 in Perlman et al. (1998), who reported a faint and quite broad $\mathrm{H} \alpha$ emission line (restframe $W_{\lambda}=30.3 \AA, F W H M=4000 \mathrm{~km} \mathrm{~s}^{-1}$ ), and classified it as a Flat Spectrum Radio Quasar (FSRQ) with $z=0.270$. Nevertheless, we have reported here our observational results for this source, since it was a candidate when we performed the observations. It presents (Fig. 5) a one-sided pc-scale jet oriented towards the northeast, changing from a PA of $\sim 50^{\circ}$ at 5 mas from the core (EVN image) to $20^{\circ}$ up to 200 mas, at MERLIN scales.

Model fitting of the EVN visibilities with circular Gaussians reveals a compact core $(0.7$ mas FWHM) with $245.4 \mathrm{mJy}$, and two distinct components, one with $11.9 \mathrm{mJy}$ at 6.2 mas (PA $46^{\circ}, 1.9$ mas $F W H M$, present in the right panel image of Fig. 5) and another one with the size of the beam, a flux density of $1.7 \mathrm{mJy}$ at a distance of 29.6 mas in PA $27^{\circ}$ (visible in the middle panel image of Fig. 5).

Using again the fact that we do not detect a counter-jet, we can use Eq. (3) with $S_{\mathrm{a}}=11.9 \mathrm{mJy}$ (the closest component to the core), $3 \sigma=0.45 \mathrm{mJy}, \alpha=0.07 \pm 0.02$ (see Paper I), and $k=3$ to be consistent with the discrete nature of the components, to obtain $\beta \cos \theta>0.51 \pm 0.04$. Hence, an upper limit 
Table 4. Summary of the obtained results after the VLA and optical observations (Paper I), and the EVN and MERLIN observations reported here. An asterisk indicates a non-expected behaviour for microquasars.

\begin{tabular}{|c|c|c|c|c|c|c|c|c|}
\hline \multirow[t]{2}{*}{ 1RXS name } & \multicolumn{2}{|l|}{ VLA obs. } & \multicolumn{2}{|c|}{ Optical obs. } & \multicolumn{3}{|c|}{ EVN+MERLIN obs. } & \multirow[t]{2}{*}{ Notes } \\
\hline & Structure & $\alpha$ & Structure & $I \mathrm{mag}$ & Structure & $\beta$ & $\theta\left[^{\circ}\right]$ & \\
\hline J001442.2+580201 & compact & -0.2 & point-like & 19.9 & two-sided jet & $>0.20$ & $<78$ & Promising candidate \\
\hline J013106.4+612035 & compact & -0.1 & point-like & 17.9 & one-sided jet & $>0.31$ & $<72$ & Promising candidate \\
\hline J042201.0+485610 & compact & $+1.6^{*}$ & extended ${ }^{*}$ & 17.5 & not detected ${ }^{*}$ & - & - & Thermal source? \\
\hline J062148.1+174736 & compact & +0.1 & extended ${ }^{*}$ & 17.6 & compact & - & - & Galaxy? \\
\hline J072259.5-073131 & one-sided jet* & -0.2 & point-like & 16.8 & bent one-sided jet* & $>0.29$ & $<73$ & Blazar? \\
\hline J072418.3-071508 & compact & +0.1 & point-like & 17.2 & bent one-sided jet* & $>0.51$ & $<60$ & Quasar (FSRQ) \\
\hline
\end{tabular}

of $\theta<60 \pm 3^{\circ}$ and a lower limit of $\beta>0.51 \pm 0.04$ is obtained, pointing towards relativistic radio jets as the origin of the elongated radio emission present in the images of this already identified quasar.

\section{Summary}

We have presented EVN and MERLIN observations of the six sources studied by Paredes et al. (2002) in their search for microquasar candidates at low galactic latitudes. The first one, namely 1RXS J001442.2+580201, displays a two-sided radio jet, which after analysis implies $\beta>0.20 \pm 0.02$ and $\theta<78 \pm 1^{\circ}$. 1RXS J013106.4+612035, displays a one-sided radio jet, requiring $\beta>0.31 \pm 0.05$ and $\theta<72 \pm 3^{\circ}$. The third

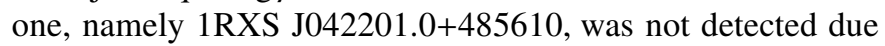
to its low flux density and/or to phase-referencing problems. 1RXS J062148.1+174736 appeared compact at all scales. The fifth one, namely 1RXS J072259.5-073131, displays a bent one-sided radio jet, implying $\beta>0.29 \pm 0.05$ and $\theta<73 \pm 3^{\circ}$. Finally, 1RXS J072418.3-071508 shows also a bent one-sided jet, requiring $\beta>0.51 \pm 0.04$ and $\theta<60 \pm 3^{\circ}$.

After a detailed analysis of our data, we show in Table 4 a summary of the results obtained after the VLA and optical observations (Paper I), and the EVN+MERLIN observations reported here. As can be seen, the first two sources, 1RXS J001442.2+580201 and 1RXS J013106.4+612035, are promising microquasar candidates. 1RXS J042201.0+485610 is probably of thermal nature due to the highly inverted spectrum at high radio frequencies, while 1RXS J062148.1+174736 is probably an extragalactic object due to the extended nature of the optical counterpart. 1RXS J072259.5-073131 shows properties common to blazars, while 1RXS J072418.3-071508 is an already identified quasar. We note that 1RXS J072259.5-073131 is bright enough at radio wavelengths to attempt an $\mathrm{H}_{\mathrm{I}}$ absorption experiment, that could allow to determine if this source is galactic or not. In any case, optical spectroscopic observations of the first five sources are in progress, to clearly unveil their galactic or extragalactic nature.

Acknowledgements. We acknowledge R. Porcas and W. Alef for their useful comments and suggestions after reading through a draft version of this paper. We acknowledge useful comments from L. F. Rodríguez, the referee of this paper. We are very grateful to S. T. Garrington, M. A. Garrett, D. C. Gabuzda, and C. Reynolds for their valuable help in the data reduction process. This paper is based on observations with the 100-m telescope of the MPIfR (Max-Planck-Institut für Radioastronomie) at Effelsberg. We thank the staff of the JIVE correlator and of the observing telescopes, especially A. Kraus for the single dish flux density measurements at the $100 \mathrm{~m}$ antenna in Effelsberg. The European VLBI Network is a joint facility of European, Chinese and other radio astronomy institutes funded by their national research councils. MERLIN is operated as a National Facility by the University of Manchester at Jodrell Bank Observatory on behalf of the UK Particle Physics \& Astronomy Research Council. The EVN observations were carried out thanks to the TMR Access to Largescale Facilities programme under contract No. ERBFMGECT950012. Part of the data reduction was done at JIVE with the support of the European Community - Access to Research Infrastructure action of the Improving Human Potential Programme under contract No. HPRICT-1999-00045. M.R., J.M.P. and J.M. acknowledge partial support by DGI of the Ministerio de Ciencia y Tecnología (Spain) under grant AYA2001-3092, as well as partial support by the European Regional Development Fund (ERDF/FEDER). During this work, M.R. has been supported by two fellowships from CIRIT (Generalitat de Catalunya, ref. 1998 BEAI 200293 and 1999 FI 00199). J.M. has been aided in this work by an Henri Chrétien International Research Grant administered by the American Astronomical Society, and has been partially supported by the Junta de Andalucía. This research has made use of the NASA's Astrophysics Data System Abstract Service, of the SIMBAD database, operated at CDS, Strasbourg, France, and of the NASA/IPAC Extragalactic Database (NED) which is operated by the Jet Propulsion Laboratory, California Institute of Technology, under contract with the National Aeronautics and Space Administration.

\section{References}

Browne, I. W. A., Wilkinson, P. N., Patnaik, A. R., \& Wrobel, J. M. 1998, MNRAS, 293, 257

Castro-Tirado, A. J., Greiner, J., \& Paredes, J. M. 2001, Microquasars, Proc. of the Third Microquasar Workshop on Galactic Relativistic Jet Sources (Kluwer Academic Publishers, Dordrecht, The Netherlands), Ap\&SS, 276

Condon, J. J., Cotton, W. D., Greisen, E. W., et al. 1998, AJ, 115, 1693

Douglas, J. N., Bash, F. N., Bozyan, F. A., Torrence, G. W., \& Wolfe, C. 1996, AJ, 111, 1945

Griffith, M. R., Wright, A. E., Burke, B. F., \& Ekers, R. D. 1994, ApJS, 90, 179

Hannikainen, D., Campbell-Wilson, D., Hunstead, R., et al. 2001, in Proc. of the Third Microquasar Workshop Galactic Relativistic Jet Sources, ed. A. J. Castro-Tirado, J. Greiner, \& J. M. Paredes (Kluwer Academic Publishers), Ap\&SS, 276, 45

Kraus, A. 1997, Ph.D. Thesis, Friedrich-Wilhelms-Universität Bonn, Germany 
Liu, Q. Z., van Paradijs, J., \& van den Heuvel, E. P. J. 2000, A\&AS, 147,25

Liu, Q. Z., van Paradijs, J., \& van den Heuvel, E. P. J. 2001, A\&A, 368,1021

Massi, M., Ribó, M., Paredes, J. M., Peracaula, M., \& Estalella, R. 2001, A\&A, 376, 217

Massi, M., Ribó, M., Paredes, J. M., et al. 2002, Sub-arcsecond radio jets in the high mass X-ray binary LS I +61 303, in Proc. of the 6th European VLBI Network Symp., ed. E. Ros, R. W. Porcas, A. P. Lobanov, \& J. A. Zensus (Max-Planck-Institut für Radioastronomie, Bonn, Germany), 279

Mioduszewski, A. J., Rupen, M. P., Hjellming, R. M., Pooley, G. G., \& Waltman, E. B. 2001, ApJ, 553, 766

Mirabel, I. F., \& Rodríguez, L. F. 1999, ARA\&A, 37, 409
Paredes, J. M., Martí, J., Ribó, M., \& Massi, M. 2000, Science, 288, 2340

Paredes, J. M., Ribó, M., \& Martí, J. 2002, A\&A, 394, 193 (Paper I)

Patnaik, A. R., Browne, I. W. A., Wilkinson, P. N., \& Wrobel, J. M. 1992, MNRAS, 254, 655

Peng, B., Kraus, A., Krichbaum, T. P., \& Witzel, A. 2000, A\&A, 145, 1

Perlman, E. S., Padovani, P., Giommi, P., et al. 1998, AJ, 115, 1253

Stirling, A. M., Spencer, R. E., de la Force, C. J., et al. 2001, MNRAS, 327,1273

Shepherd, M. C., Pearson, T. J., \& Taylor, G. B. 1994, BAAS, 26, 987

Voges, W., Aschenbach, B., Boller, Th., et al. 1999, A\&A, 349, 389

Wagner, S. J., \& Witzel, A. 1995, ARA\&A, 33, 163 